\title{
An RL-Based Approach for IEQ Optimization in Reorganizing Interior Spaces for Home-Working
}

\author{
Patrizia RIBINO ${ }^{\mathrm{a}, 1}$, and Marina BONOMOLO ${ }^{\mathrm{b}}$ \\ ${ }^{a}$ Istituto di Calcolo e Reti ad Alte prestazioni (ICAR), National Research Council, Italy \\ ${ }^{\mathrm{b}}$ Dipartimento di Ingegneria, Universita degli Studi di Palermo
}

\begin{abstract}
Indoor Environmental Quality (IEQ) is a very important aspect in the design of spaces. It depends on fundamental requirements, and it aims to improve living quality and ensure high well-being for the occupants. Poor IEQ has significant health consequences as people spend a considerable portion of their time indoors. The restrictions imposed by the current SARS-CoV-2 pandemic have worsened this situation by requiring people to spend more time at home and adopt home-working as a primary work model. Such a situation requires individuals to reconfigure their interior home spaces for adapting to new emerging needs. This paper proposes an approach based on reinforcement learning to support the rearrangement of indoor spaces by maximizing the indoor environmental quality index in terms of thermal, acoustic and visual comfort in the new furniture layout scheme.
\end{abstract}

Keywords. Indoor Environmental Quality, Reinforcement Learning, Interior Layout Design

\section{Introduction}

People spend most of their time in buildings [1]. It is estimated that individuals spend more than $80 \%$ of the day indoors. The maintenance of indoor environmental quality (IEQ) is therefore significant for improving occupants' feeling of comfort, health, working efficiency, and productivity[2]. Nowadays, this requirement is becoming more relevant due to the pandemic emergency caused by the SARS-CoV-2. The extremely contagious nature of the virus and the severity of the Covid-19 disease have resulted in strict stay-in-home and social distancing policies in several countries across the world. In particular, to contain the spread of the coronavirus and curb contagion, home working ${ }^{2}$ is becoming the most common model of work. Such a new situation leads people to rearrange some home spaces and to reconfigure new furniture layout schemes to be comfortable, as much as possible, in their home-work. Thermal, visual and acoustic conditions are the key elements that jointly influence indoor comfort [2]. Many standards [3] and

\footnotetext{
${ }^{1}$ Corresponding Author: Istituto di Calcolo e Reti ad Alte prestazioni (ICAR), via Ugo La Mafa 113, Palermo, Italy; E-mail: patrizia.ribino@icar.cnr.it.

${ }^{2}$ In this work, we prefer to use the term home-working rather than the widely used term of 'smart working' because this latter is not constrained to be performed strictly at home as the Covid-19 restrictions impose.
} 
studies propose methods and indexes to evaluate the quality of the environment. They indicate methods and criteria for designing indoor environments related to the internal air quality, illuminance level, and thermal and acoustic parameters. In general, such standards are based on measurable values directly collected from the environment employing appropriate sensors. Although several existing works deal with the interior automatic layout design, they do not consider IEQ issues. To cite a few, Yang et al. [4] present a learning-based solution for an automatic interior layout that considers user-specified furniture to be placed in appropriate functional areas. Liu et al. [5] reconstructed 3D scenes from photos capturing different orientations. Yu et al. [6] present a method based on energy function for the overall layout by considering relations between furniture and the room function, introducing layout constraints.

Moreover, in recent years, extensive research efforts have been spent on the development of artificial intelligence (AI) techniques, particularly in contexts where individual well-being is a key element, such as ambient assisted living (AAL) [7, 8], health-care $[9,10]$ and intelligent environments [11]. Among AI techniques, reinforcement learning [12] plays an increasingly important role in achieving specific goals in unknown environments by adopting a trial-and-error paradigm.

The contribution of this paper is a novel method for generating optimal layout configurations in terms of environmental comfort conditions. In particular, we propose the use of Reinforcement Learning techniques for an automatic and intelligent furniture layout generation of a given room with office end-use that maximizes the environmental comfort of an individual in performing his/her working activities. In particular, we formulated the interior layout problem as a Markov decision process by defining states, actions, and reward function according to the constraints given by the IEQ standards.

A pilot study about a home office configuration is presented. Preliminary results give evidence that the proposed IEQ-based RL approach could be a valuable tool for an automatic indoor design that evaluates the conformity to the standard levels of environmental comfort during the design phase. Indeed, a common method called Post Occupancy Evaluation $[13,14]$ is used to evaluate an occupant's comfort conditions (after (s)he lived in that space) and make some successive modifications to reach desired occupant comfort conditions. The proposed approach allows evaluating a priori the best comfortable conditions that can be reached by an environment endowed with specific equipment. The current version of the software prototype is realized for finding optimal configurations of room with office end-use. However, it can be extended as a generalized tool for dealing with different functional environments and furniture.

The rest of the paper is organized as follows. Section 2 introduces a theoretical background of IEQ and RL. Section 3 presents the RL approach for generating comfortable indoor layouts. Section 4 shows a pilot study about a rearrangement of home space for working destination. Finally, in Section 5, conclusions and future works are shown.

\section{Theoretical Background}

\subsection{Indoor comfort conditions}

The primary contribution to IEQ satisfaction is linked to Thermal, Visual and Acoustics Comfort and Air Quality [2]. In this paper, we consider the first three. 
Thermal Comfort - The first model of thermal comfort was established by Fanger in the 1970s [15]. Predicted Mean Vote (PMV), as a result of Fanger's comfort equation, predicts the mean response of a larger group of people according to the ASHRAE thermal sense scale [15]. PMV depends on a combination of environmental and individual variables $P M V=f\left(t_{a}, t_{m r}, v_{a}, p_{v}, M, I_{c l}\right)$ where $t_{a}$ is the air temperature $\left({ }^{\circ} C\right), t_{m r}$ is the mean radiant temperature $\left({ }^{\circ} \mathrm{C}\right), v_{a}$ is the relative air velocity $(\mathrm{m} / \mathrm{s}), p_{v}$ is the water vapor partial pressure $(\mathrm{Pa}), \mathrm{M}$ is the metabolic rate $\left(\mathrm{W} / \mathrm{m}^{2}\right)$, and $I_{c l}$ is the clothing insulation $\left(m^{2} K / W\right)$. PMV can be expressed in a concise form as:

$$
P M V=\left(0.303 \times \exp ^{-0.036 M}+0.028\right) \times L
$$

where $\mathrm{L}$ is the thermal load on the body. It is the difference between the rate of metabolic heat generation and the calculated heat loss from the body to the actual environmental conditions assumed as optimum at a given activity level [15]. The metabolic rate $(M)$ mainly corresponds to the heat production of the human body. Using PMV, the Percentage of People Dissatisfied (PPD) can be predicted. PPD indicates the number of people dissatisfied with the thermal environment and it is determined as follows:

$$
P P D_{T C}=100-95 \times \exp \left(-0.03353 * P M V^{4}-0.2179 \times P M V^{2}\right)
$$

Depending on the ranges of PPD and PMV, three kinds of comfort zones can be identifies[16], as shown in Table 1. Category A corresponds to a high-performance building, while category $\mathrm{B}$ and category $\mathrm{C}$ corresponds respectively to a medium and moder-

\begin{tabular}{|c|c|c|c|}
\hline \multirow{2}{*}{ Parameters } & \multicolumn{3}{|c|}{ Recommended Limits } \\
\hline & Category & Summer & Winter \\
\hline \multirow{3}{*}{ PMV } & $\mathrm{A}$ & \multicolumn{2}{|c|}{$-0.2<\mathrm{PMV}<0.2$} \\
\hline & B & \multicolumn{2}{|c|}{$-0.5<\mathrm{PMV}<0.5$} \\
\hline & $\mathrm{C}$ & \multicolumn{2}{|c|}{$-0.7<\mathrm{PMV}<0.7$} \\
\hline \multirow{3}{*}{ PPD, [\%] } & A & \multicolumn{2}{|c|}{$<6$} \\
\hline & B & \multicolumn{2}{|c|}{$<10$} \\
\hline & $\mathrm{C}$ & \multicolumn{2}{|c|}{$<15$} \\
\hline
\end{tabular}
ate one. In this study, we refer to category B.

Table 1. Thermal comfort according to PPD and PMV.

Visual Comfort - Visual comfort is related to the quantity and quality of light within a given space at a given time. Light is a fundamental element in our capacity to see. Both too little and too much light can cause visual discomfort. Visual comfort mainly depends on photo-metric quantities such as the illuminance (i.e., the amount of luminous flux per unit area), luminance and, consequently, glare condition and contrast. Further aspects, such as views of outside space or light quality, are also considered. As concerns, the visual comfort, the European standard EN 12464-1[17] defines lighting requirements for indoor work areas in terms of quantity and quality of illumination. It covers offices, places of public assembly, restaurants/hotels, theaters/cinemas. For the aim of this paper, we refer to the standard for generic offices that recommends $500 l x$ on the work-plane. 


\begin{tabular}{|c|c|c|c|}
\hline Domain & Room/Space & NR Value & $\begin{array}{l}\text { Recommended Noise Level } \\
\text { (dB) }\end{array}$ \\
\hline \multirow[t]{2}{*}{ Industrial } & Control Rooms & $40-50$ & $45-55 \mathrm{~dB}(\mathrm{~A})$ \\
\hline & Offices & $35-40$ & $40-45 \mathrm{~dB}(\mathrm{~A})$ \\
\hline \multirow{2}{*}{$\begin{array}{l}\text { Commercial } \\
\text { and Leisure }\end{array}$} & Offices & $35-40$ & $40-45 \mathrm{~dB}(\mathrm{~A})$ \\
\hline & Private Offices & $30-35$ & $35-40 \mathrm{~dB}(\mathrm{~A})$ \\
\hline
\end{tabular}

Table 2. Recommended noise levels.

Acoustic Comfort - Providing acoustic comfort consists of minimizing noise and maintaining satisfaction among residents. Acoustic comfort helps to improve concentration. Hence, buildings are designed to specific noise standards based on their use. For example, the noise level in a library may differ from noise specifications in a public hall. Noise Ratings (NR) [18] is one of the standards used to evaluate acoustic comfort. Each space or room has a recommended NR value, which is based upon the intended requirements, needs and function of the indoor environment. Table 2 shows an excerpt of the recommended NR levels for some application areas and the related mapping with the most common unit for measuring sound pressure level, the $\mathrm{dB}$ level.

\subsection{Reinforcement Learning}

Reinforcement Learning (RL) is a branch of machine learning inspired by behaviorist psychology, where human and animal behavior is studied from a reward and punishment perspective. According to this perspective, an RL approach concerned with how an autonomous agent, that senses and acts in a dynamic environment, learns to choose the most effective actions to achieve its goals by maximizing its overall rewards[12]. The agent learns the optimal control policy by trails during the interactions with the environment. This agent-environment interaction process is most commonly formulated as a Markov Decision Process (MDP), consisting of State, Action, and Rewards. One of the most popular learning algorithms is Q-learning [19]. Q-learning's objective is to find an optimal policy in the sense that the expected value of the total reward overall successive steps is the maximum achievable.

The core of the Q-learning algorithm is the Bellman Equation (see eq.3), according to which the optimal Q-value for a given state-action pair is equal to the sum of the maximum reward the agent can get from an action in the current state and the maximum discounted reward it can obtain from any possible state-action pair that follows.

$$
Q\left(s_{t}, a_{t}\right) \leftarrow Q\left(s_{t}, a_{t}\right)+\alpha \times\left[R+\gamma \times \max _{a_{t+1}} Q\left(s_{t+1}, a_{t+1}\right)-Q\left(s_{t}, a_{t}\right)\right]
$$

where $\mathrm{R}$ is the reward received when an agent moves from the state $s_{t}$ to the state $s_{t+1}$. The learning rate $\alpha$ determines to what extent newly acquired information overrides old information. The discount factor $\gamma$ determines the current value of future rewards.

\section{IEQ-based Q-Learning approach for organizing indoor space}

In this section, we present the proposed RL approach for the optimization of indoor space configurations. In particular, such an optimized configuration is obtained using the Q-learning method driven by IEQ standards. 


\subsection{Problem Formulation}

A practical furniture layout scheme has to meet functional requirements and respects some primary layout constraints (e.g., doors and windows cannot be impeded and furniture has to be reachable). In this paper, we consider interior layout design as the problem to find the best furniture layout that provides the most comfortable configuration for an area with office end-use in terms of thermal, acoustics and visual comfort. Moreover, we assume that a set $F$ of furniture for equipping the room is selected by the occupant.

We express the problem of IEQ-based indoor layout configuration as a Markov Decision Process defined by the following tuple:

$$
M D P=<S, S_{0}, S_{f}, F, \operatorname{Act}(.), P, R>
$$

In particular, $S$ is a finite set of states of the world represented by different furniture locations. $S_{0} \in S$ is an initial state of the world. It is represented by an initial placement of furniture on the room plane. $S_{f} \in S$ is a final state characterized by an optimal IEQ. $F$ is the set of user furniture to be positioned in a room. Act $(f)$ is a finite set of possible movements for each furniture $f \in F$. In this paper, $\operatorname{Act}(f)=$ $\{$ moveU p, moveDown, moveRight, moveLeft $\}$. $P$ is a probability distribution function. When a movement of an item is executed, the world makes a transition from its current state $S$ to a succeeding state $S^{\prime}$. The probability of this transition is labeled as $P\left(S^{\prime} \mid S, a\right)$. $R\left(S^{\prime} \mid S, a\right)$ is the immediate reward obtained by moving a piece of furniture from a current position to a new position.

Let assume $n$ different pieces of furniture $\left(f_{1}, f_{2}, \ldots, f_{n}\right)$. Our aim is to place these objects onto some locations $\left(l_{1}, l_{2}, \ldots l_{n}\right)$. We use RL to find a mapping $\left(f_{1}, f_{2}, \ldots, f_{n}\right) \rightarrow$ $\left(l_{1}, l_{2}, \ldots l_{n}\right)$ that maximizes a reward function $\mathrm{R}$ subject to some constraints. Mainly, there is a unique location for placing each object $f_{i}$, but each location $l_{j}$ can be assigned to any furniture piece. The proposed RL approach's objective is to find an optimal distribution of furniture on several locations such that it maximizes the rewards (in terms of thermal, acoustics and visual comfort) generated from each configuration.

For the sake of simplicity, we considered an indoor space as a rectangular room with predefined dimensions. A reference system is associated with the room plant, whose origin corresponds to the top left corner. Each piece of furniture has a location defined by a couple of coordinates $(x, y)$ that corresponds to the top left corner of the furniture and size $s$ represented by a couple (width,depth). In a room, several kinds of objects may be located. Each step, the RL agent places a furniture $f_{j}$ at a new location $l_{j}=\left(x_{j}, y_{j}\right)$. It is worth noting that some types of furniture can influence the sound pressure level, temperature, and environment's illuminance. Hence, after the agent action, the reward is evaluated considering the effect of the furniture displacement on comfort conditions.

In this paper, we address the indoor layout problem as the problem to configure a home office with office furniture (i.e., desk, printer, heater) to create a comfortable indoor space for home-working. In particular, the reward function is evaluated according to the desk's location, considered as the reference point. Indeed, we assume that the worker spends much of his/her working time in that position.

\subsection{Reward Function}

The reward function is related to the Indoor Environment Quality index, which is considered, in the most prominent literature, as an indicator of the level of comfort. IEQ index 
refers to the building's indoor environment quality considering the occupants' satisfaction level presented on a $0-100 \%$ scale (as a percentage of users satisfied). It is composed of four indoor comfort sub-indexes (also presented on a $0-100 \%$ scale): thermal comfort $T C_{\text {index }}$, acoustic comfort $A C_{\text {index }}$, visual comfort $V C_{\text {index }}$ and indoor air quality $I A Q_{\text {index }}$ index. Some weights are associated with these indexes for the evaluation of the whole IEQ index. In this paper, we refer to the IEQ model proposed in [20]. Moreover, in this preliminary study, we do not consider the effect of the indoor air quality index on the IEQ. Hence, we formulated the reward function of the proposed RL approach as a weighted average of the comfort indexes as mentioned earlier:

$$
R_{I E Q}=w_{1} * T C_{\text {index }}+w_{2} * A C c_{\text {index }}+w_{3} * V C_{\text {index }}
$$

$T C_{\text {index }}$ refers to the percentage of people accepting the thermal environment:

$$
T C_{\text {index }}=100-P P D_{T C}
$$

where $P P D$ is the Predicted Percentage Dissatisfied calculated according to Eq.2. $A C_{\text {index }}$ relates to the building's ability to provide an environment with minimal unwanted noise. We assume that this noise source may be external or internal to the indoor space we are considering. Internal noise, for example, can be generated by an HVAC (Heating, Ventilation and Air Conditioning) system. Conversely, external noise can be propagated, for example, by a home appliance placed in a space adjacent to the room under study. According to the IEQ model [20], the $A C_{\text {index }}$ is given by:

$$
A C_{\text {index }}=100-P D_{\text {Acc }}
$$

where $P D_{A c c}$ is the predicted percentage of sound level dissatisfied occupants with the change in noise level from Recommended to Actual value on a $0-100 \%$ scale:

$$
P D_{\text {Acc }}=2 *\left(\text { Actual }_{\text {SoundPressureLevel }}-\text { Reccomended }_{\text {SoundPressureLevel }}\right)
$$

Moreover, to evaluate the $A C_{\text {index }}$, the noise reduction due to the source's distance to the receiver has to be considered. For a continuing sound source in a room, the sound level is the sum of direct and reverberant sound [21]. The sound pressure level for a receiver is given by:

$$
L_{\text {Receiver }}=L_{\text {Source }}+10 \log \left(Q /\left(4 \pi r^{2}\right)+4 / R\right)
$$

where $L_{\text {Receiver }}$ is the sound pressure level $(\mathrm{dB})$ received in a given point of the room at $r$ distance from the source. $L_{\text {Source }}$ is the sound power level from the source of the noise. $Q$ is the directivity factor that measures the directional characteristic of a sound's source. It assumes predefined values for the specific location of the sound source. Finally, the room constant $R$ expresses the acoustic property of a room according to:

$$
R=S \bar{\alpha}_{a b s} /\left(1-\bar{\alpha}_{a b s}\right)
$$

where $S$ is the total surface of the room (including walls, floor and ceiling) and $\bar{\alpha}_{a b s}$ is the mean absorption coefficient of the room.

$$
\bar{\alpha}_{a b s}=\frac{\sum_{i=1}^{n} S_{i} \alpha_{i}}{\sum_{i=1}^{n} S_{i}}
$$


where $S_{i}$ is an individual surface in the room $\left(\mathrm{m}^{2}\right)$ made with a specific material that is characterized by an absorption coefficient $\alpha_{i}{ }^{3}$. Moreover, we assume that several sources of noise can affect the environment. Thus, we have to consider each source's contribution to the receiver given by eq eq. 12 with $k$ is the number of sources and $L_{j}$ is the sound pressure level of the $j^{\text {th }}$ source.

$$
L_{\text {TotReceiver }}=10 * \log \sum_{j=1}^{k} 10^{0.1 * L_{j}}
$$

Finally, the visual comfort index $V C_{\text {index }}$ is based on the amount of light falling on the working plane as follows:

$$
V C_{\text {index }}=100-P D_{L}
$$

where $P D_{L}$ is the percentage of persons dissatisfied with minimum daylight or the probable percentage of people switching on artificial lighting on a scale of $0-100 \%$. The predicted percentage of dissatisfied occupants is calculated as a function of daylight luminance $E_{\min }$ with the following equation:

$$
P D_{L}=\frac{(-0.0175+1.0361)}{1+\exp \left[4.0835 \times\left(\log \left(E_{\min }\right)-1.8223\right)\right.} \times 100
$$

On the other hand, according to the interior design principles, we also considered the following layout rules for indoor space dedicated to office end-use:

1. make the desk near the window. Such rule allows having more natural light on the desk, but at the same time, it satisfies IEQ constraints about visual comfort that, as previously said, is also improved by views of outside space;

2. make the furniture far from the door for not impeding its regular use.

Two reward functions $R_{\text {window }}$ and $R_{\text {door }}$ associate a quantitative measure to the compliance with rule 1 and 2, respectively. Hence, the total reward is given by:

$$
R_{T o t}=R_{I E Q}+R_{\text {window }}+R_{\text {door }}
$$

\subsection{IEQ-based Q-Learning Algorithm}

The pseudo-code of the IEQ-based Q-Learning algorithm is shown in Algorithm 1. Starting from the MDP model and the characteristics of the room to be configured, Algorithm 1 provides a mapping of the furniture $\left(f_{1}, f_{2}, \ldots, f_{n}\right)$ to some room locations $\left(l_{1}, l_{2}, \ldots l_{n}\right)$ that maximizes the environmental comfort of the occupant. Firstly, Algorithm 1 initializes a matrix representing the room under study. For each furniture $f_{i}$ a set of available actions $\operatorname{Act}\left(f_{i}\right)$ is available. Then, Algorithm 1 randomly selects the initial positions of the furniture that have been located in the room and initializes the Q-learning matrix. An initial reward is calculating for the initial state $S_{0}$ according to Eqs. 5 - 15. For each episode of the learning process, starting from the initial state $S_{0}$, the q-learning agent chooses an action from the list of the available actions that allows moving furniture in the selected action direction. The exploration strategy used in this paper is the $\varepsilon$-greedy policy. Hence, a new reward is evaluated for the new observed state $S^{\prime}$ according to Eqs. 5 - 15, and the Q-learning matrix is updated accordingly. An optimal final state is a state

\footnotetext{
${ }^{3}$ Common absorption coefficients can be found at https://www.acoustic.ua/st/web_absorption_data_eng.pdf
} 


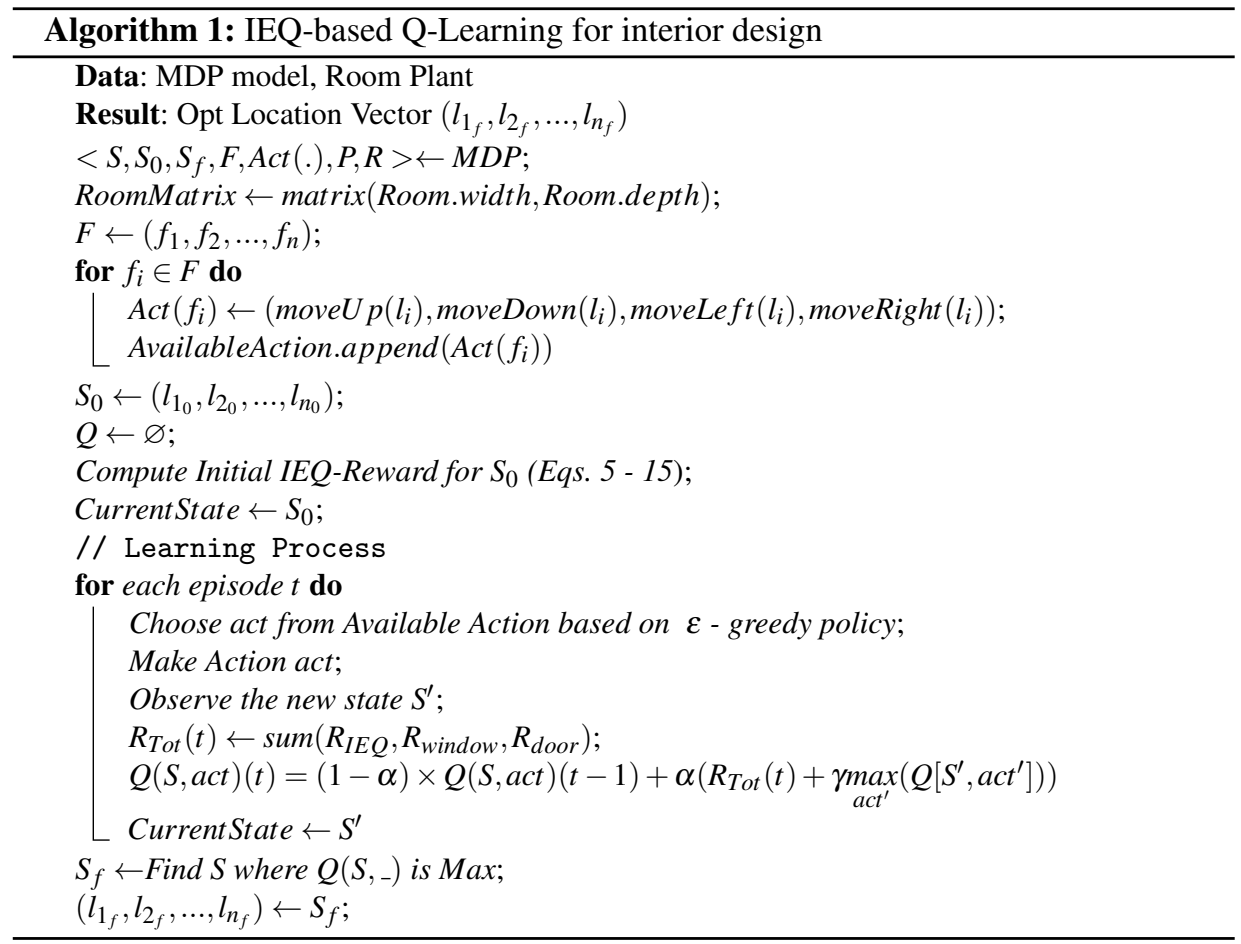

having a max q-learning value. Hence, the mapping of the furniture on the locations of the room that maximizes the environmental comfort for that room and respects the layout design constraints is given by $\left(f_{1}, f_{2}, \ldots, f_{n}\right) \leftarrow\left(l_{1_{f}}, l_{2_{f}}, \ldots, l_{n_{f}}\right)$

\section{A pilot study on a simulated home office}

To perform a preliminary evaluation of the proposed approach, we considered a simple case study built on a simulated room. A desk and a portable heater must be put for realizing a comfortable home office in such a room. The size of the simulated room is $4 \times$ $3 \times 3 \mathrm{~m}$. The room plant is represented as a grid where the dimension of each cell is $0.5 \times$ $0.5 \mathrm{~m}$. The room has 3 windows (yellow rectangles $W$ ) and a door (blue rectangle $D$ ) located as it is shown in Fig. 1. The room has $12 \mathrm{~m}^{2}$ of parquet on the floor with absorption coefficient $\alpha_{a b s}=0.2,12 \mathrm{~m}^{2}$ of roof with absorption coefficient $\alpha_{a b s}=0.1,9 \mathrm{~m}^{2}$ of large panes made of $4 \mathrm{~mm}$ glass with absorption coefficient $\alpha_{a b s}=0.3$ and $33 \mathrm{~m}^{2}$ of walls with absorption coefficient $\alpha_{a b s}=0.1$. The portable heater produces a noise of $50 \mathrm{~dB}$, and, for this study, a setpoint temperature of $23^{\circ} \mathrm{C}$ has been supposed. In the adjacent space outdoor of the room near the door, there is a domestic appliance (i.e. a washing machine) that produces a noise of $50 \mathrm{~dB}$. The occupant's metabolic rate for typical office task is 1.2 met and we assume a medium clothes thermal insulation $I_{c l}=1.0$ clo for winter season and an air velocity $v_{a}=0.1 \mathrm{~m} / \mathrm{s}$. The relative humidity is $p_{v}=50 \%$. Acquisition of daylight interior illuminance values is obtained by the lighting simulation software Dialux Evo [22]. In particular, the room's central space is characterized by illuminance 

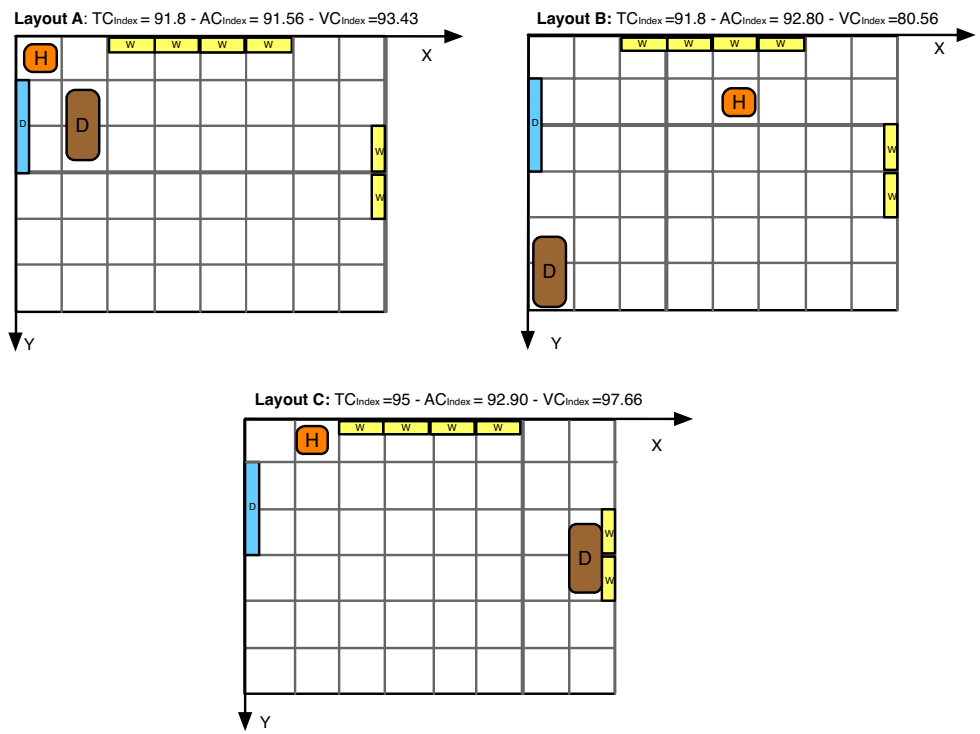

Figure 1. Layout configurations with different comfort conditions.

values around $300 l x$, the area close to the windows shows about $500 l x$. The area near the bottom left corner of the room has the lowest illuminance level (i.e., 150lx). According to the IEQ standards, for the case under study, we consider as acceptable values of comfort index the following: $T C_{\text {index }}>90, V C_{\text {index }}>97$ and $A C_{\text {index }}>95$.

In Fig.1, we show some three layout configurations at three different steps of the algorithm. Layout $A$ shows an initial configuration of the room with the portable heater on the top left corner and the desk near the door. This furniture layout provides to an occupant that works at the desk the following index of comfort: $T C_{\text {index }}=91.8, A C_{\text {index }}=$ 91.56 and $V C_{\text {index }}=93.43$. Layout $B$ shows an intermediate step of the algorithm where the desk is on the bottom left corner of the room and the portable heater is near the windows of the opposite wall. In this case, we can see that $T C_{\text {index }}=91.8, A C_{\text {index }}=$ 92.80 and $V C_{\text {index }}=80.56$. At it is expected, the low value of the $V C_{\text {index }}=80.56$ is determined by the low illuminance level at the desk location. Finally, Layout $C$ shows an optimal result obtained by the algorithm. In this case, the IEQ indexes show better values compared with the previous ones. In this state, the desk's location is near the window on the vertical wall, and the location of the portable heater is near the top left corner. We can see that $T C_{\text {index }}=95, A C_{\text {index }}=92.90$ and $V C_{\text {index }}=97.66$. Since the standard for acoustics recommends $45 \mathrm{~dB}$, the desk's distance from the portable heater and the door is not enough to reduce the combined effect of the two sources of noise at the recommended value. Thus, we cannot have a notable improvement in acoustic comfort for this room with the kind of furniture understudy. For this reason, the $A C_{\text {index }}$ does not reach an adequate value. Conversely, a significant improvement is shown in the visual and thermal comfort determined by the windows' proximity, showing high illuminance values and better operative temperature. As we can see, the optimal configuration also respects the layout design constraints, as previously mentioned. 


\section{Conclusions}

The paper presents an approach for reorganizing interior spaces for home-working. The problem is addressed using RL techniques to obtain optimized layout configuration in terms of Thermal, Visual and Acoustic comfort. Although the work is still preliminary, the results of some tests are encouraging. We are programming to conduct an extensive set of tests on different real cases and develop some software functionality that will allow the process to be automatized entirely without any external calculation and to cope with different kinds of environments.

\section{References}

[1] D. Mumovic, M. Santamouris, A Handbook of Sustainable Building Design and Engineering:" An Integrated Approach to Energy, Health and Operational Performance", Routledge, 2013.

[2] M. Frontczak, P. Wargocki, Literature survey on how different factors influence human comfort in indoor environments, Building and environment 46 (4) (2011) 922-937.

[3] A. Standard, 55-92. thermal environmental comfort conditions for human occupancy. ashrae (1992).

[4] B. Yang, L. Li, C. Song, Z. Jiang, Y. Ling, Automatic interior layout with userspecified furniture, Computers \& Graphics 94 (2021) 124-131.

[5] Z. Liu, S. Tang, W. Xu, S. Bu, J. Han, K. Zhou, Automatic 3d indoor scene updating with rgbd cameras, in: Computer Graphics Forum, Vol. 33, Wiley Online Library, 2014, pp. 269-278.

[6] L. F. Yu, S. K. Yeung, C. K. Tang, D. Terzopoulos, T. F. Chan, S. J. Osher, Make it home: automatic optimization of furniture arrangement, ACM Transactions on Graphics (TOG)-Proceedings of ACM SIGGRAPH 2011, v. 30,(4), July 2011, article no. 8630 (4).

[7] A. Coronato, G. Paragliola, A structured approach for the designing of safe aal applications, Expert Systems with Applications 85 (2017) 1-13.

[8] A. Coronato, G. De Pietro, Situation awareness in applications of ambient assisted living for cognitive impaired people, Mobile Networks and Applications 18 (3) (2013) 444-453.

[9] A. Coronato, M. Naeem, G. De Pietro, G. Paragliola, Reinforcement learning for intelligent healthcare applications: A survey, Artificial Intelligence in Medicine 109 (2020) 101964.

[10] M. Naeem, G. Paragliola, A. Coronato, A reinforcement learning and deep learning based intelligent system for the support of impaired patients in home treatment, Expert Systems with Applications 168 (2021) 114285.

[11] A. Coronato, G. De Pietro, Tools for the rapid prototyping of provably correct ambient intelligence applications, IEEE Transactions on Software Engineering 38 (4) (2011) 975-991.

[12] M. Naeem, S. T. H. Rizvi, A. Coronato, A gentle introduction to reinforcement learning and its application in different fields, IEEE Access (2020). 
[13] J.-H. Choi, K. Lee, Investigation of the feasibility of poe methodology for a modern commercial office building, Building and Environment 143 (2018) 591-604.

[14] P. Li, T. M. Froese, G. Brager, Post-occupancy evaluation: State-of-the-art analysis and state-of-the-practice review, Building and Environment.

[15] P. O. Fanger, et al., Thermal comfort. analysis and applications in environmental engineering., Thermal comfort. Analysis and applications in environmental engineering.

[16] D. Markov, Practical evaluation of the thermal comfort parameters, Annual International Course: Ventilation and Indoor climate, Avangard, Sofia (2002) 158-170.

[17] E. UNI, 12464-1: 2011, Light and lighting. Lighting of work places. Part 1.

[18] L. L. Beranek, W. E. Blazier, J. J. Figwer, Preferred noise criterion (pnc) curves and their application to rooms, The Journal of the Acoustical Society of America 50 (5A) (1971) 1223-1228.

[19] C. J. Watkins, P. Dayan, Q-learning, Machine learning 8 (3-4) (1992) 279-292.

[20] M. Piasecki, K. Kostyrko, S. Pykacz, Indoor environmental quality assessment: Part 1: Choice of the indoor environmental quality sub-component models, Journal of Building Physics 41 (3) (2017) 264-289.

[21] H. B. Lewis, L. Bell, Industrial noise control, fundamentals and applications, new york: M (1994).

[22] DIAL, Dialux evo 8.2, professional lighting design software (2018). URL Available on line at https://www.dial.de/en/dialux-desktop/ 